\title{
LIV. On the wave-surface in the theory of double refraction
}

\section{J.W. Lubbock Esq. F.R.S.}

To cite this article: J.W. Lubbock Esq. F.R.S. (1837) LIV. On the wave-surface in the theory of double refraction, Philosophical Magazine Series 3, 11:69, 417-421, DOI: 10.1080/14786443708649318

To link to this article: http://dx.doi.org/10.1080/14786443708649318

$$
\text { 曲 Published online: } 01 \text { Jun } 2009 .
$$

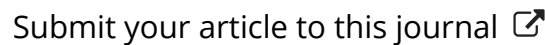

Џ Article views: 2

Q View related articles $₫$ 
the one for the other. The second objection has apparently more force, but I am able to prove that the vessels are not coated with silica, but are actually composed of silica, by showing that if the silica be removed, no trace of vessel remains. I could wish to give particular importance to the mode of working out this result, and to some of the facts connected with it, because much light appears to be thrown upon the nature of a new and beautiful portion of vegetable structure, I mean the system of; apparently, small cups, which are placed along the siliceous columns of gramineous plants*.

In order to effect the removal of silica without disturbing other parts of the plant, I placed a small portion of one of the lower leaves of a stalk of wheat for upwards of twelve hours in caustic potash. After removing the potash by dilute muriatic acid, I mounted half of the specimen in balsam, and then expelling the carbon from the other half by the aid of a spirit-lamp, I inclosed this portion also in the same substance. These I compared with each other and with the adjoining part of the leaf in its natural state. The caustic potash had effected the entire removal of the system of siliceous vessels between the ribs of the leaf, but the small cups which are duly arranged along the siliceous columns remained undisturbed. These cups, therefore, are not composed of silica; neither are they carbonaceous, for after resisting the action of potash, they resist also the action of fire. This is not the case with the ducts, \&c. which form the ribs of the leaf; they are readily carbonized and dissipated. But after the carbonaceous parts have been thus expelled, and these cups alone remain, if then a sufficient degree of heat be applied to effect their fusion, they leave upon the platinum spoon a permanent light-blue stain. It would appear, therefore, that the metallic oxides, which are always found in the ashes of wheat, exist in the plant under an, organized form, and are obtained by incineration from this system of cups. And, hence, I conclude generally that earthy, saline, and metallic ingredients enter as organizable products into the structure of plants.

Peckham, Oct. 2, 1837.

LIV. On the Wave-surface in the Theory of Double Refraction. By J. W. Luввоск, Esq., F.R.S.†

SINCE Fresnel's discovery of the wave-surface published in his admirable paper on Double Refraction (Mémoires de l'Institut, tome vii.) much has been done to elucidate this

* See the figure, Plate I. Phil. Mag., July 1837.

+ Communicated by the Author.

Third Series. Vol. 11. No.69. Nov. 1837. 
subject by eminent mathematicians; perhaps however it may not be uninteresting to return to Fresnel's reasoning through which the equation to the wave-surface was originally established, so as to facilitate the comparison of Fresnel's ideas with those which have been since developed by M. Cauchy, and by other philosophers*. With this view I have drawn up the following very brief remarks. It seems to have been generally considered that $M$. Cauchy's wave-surface is identical with that of Fresnel, but I confess that I do not think that this point has been sufficiently considered; and indeed it has already been asserted by $\mathrm{Mr}$. Kelland that little construction beyond the explanation of dispersion can be put upon M. Cauchy's results, from their great complexity.

Let the displacements of the particle $m$ in the directions of $x, y, z$ be $\xi, \eta, \zeta$, and those of $\mathfrak{m}^{\prime}, \xi+\Delta \xi, y+\Delta y, \zeta+\Delta \xi$ at the end of the time $t$, then

$$
\begin{aligned}
& \frac{\mathrm{d}^{2} \xi}{\mathrm{d} t^{2}}=\mathfrak{m} \Sigma\left\{\left(r \phi+\psi(r) \Delta x^{2}\right\} \Delta \xi+\psi r \Delta y \Delta x \Delta \eta\right. \\
& +\psi r \Delta x \Delta z \Delta \zeta\} \\
& \mathrm{d}_{t^{\mathrm{g}}}=\mathfrak{m} \Sigma\left\{\psi r \Delta y \Delta x \Delta \xi+\left\{\phi r+\psi\left(r \Delta y^{\mathrm{g}}\right\} \Delta \eta\right.\right. \\
& +\psi r \Delta y \Delta z \Delta \zeta\} \\
& \frac{\mathrm{d}^{\mathrm{g}} \zeta}{\mathrm{d} t^{2}}=\mathfrak{m} \Sigma\{\psi r \Delta x \Delta z \Delta \xi+\psi r \Delta y \Delta z \Delta \eta \\
& \left.+\left\{\phi r+\psi(r) \Delta z^{8}\right\} \Delta \zeta\right\} \text {. }
\end{aligned}
$$

These are M. Cauchy's equations, Exercises, vol. iii. p. 192: they are also given by Mr. Tovey, L. \& E. Phil. Mag., vol. viii. p. 9; and by Mr. Kelland, Cambridge Trans., vol. vi. p. 158.

The remarks of Fresnel, p. 85 to 95 , Mém. de l'Institut, vol. vii. amount to showing that these equations may, when the axes of elasticity are taken for the coordinate axes, be reduced to the form

$$
\begin{aligned}
\frac{\mathrm{d}^{2} \xi}{\mathrm{d} t^{3}} & =\mathfrak{m} \Sigma\left\{\phi r+\psi(r) \Delta x^{2}\right\} \Delta \xi \\
\frac{\mathrm{d}^{2} \eta}{\mathrm{d} t^{8}} & =\mathfrak{m} \Sigma\left\{\phi r+\psi(r) \Delta y^{2}\right\} \Delta \eta \\
\frac{\mathrm{d}^{2} \zeta}{\mathrm{d} t^{2}} & =\mathfrak{m} \Sigma\left\{\phi r+\psi(r) \Delta z^{2}\right\} \Delta \zeta .
\end{aligned}
$$

[" Prof. Powell's "Abstract of M. Cauchy's Views" appeared in Lond. and Edinb. Phil. Mag,, vol. vi. p. 16, et seq.-Eort.] 
If $\Delta \xi^{2}+\Delta \eta^{2}+\Delta \xi^{2}=\Delta \rho^{2}$, and if $X, Y, Z$ denote the angles which the direction of displacement makes with the coordinate axes,
$\Delta \xi=\Delta \rho \cos X$
$\Delta \eta=\Delta \rho \cos Y$
$\Delta \zeta=\Delta \rho \cos z$

$X, Y, Z$ being the same as in Fresnel's notation,

$$
\cos ^{2} X+\cos ^{2} Y+\cos ^{2} Z=1 \text {. }
$$

The simplest case, and I believe that implicitly intended by Fresnel, is when the amplitude $\Delta \rho$ depends only upon the distance $r$ from the origin, then

$$
\begin{gathered}
\Delta \rho=\frac{\mathrm{d} \rho}{\mathrm{d} r} \Delta r+\frac{\mathrm{d}^{2} \varrho}{1.2 \mathrm{~d} r^{2}} \Delta r^{2}+\frac{\mathrm{d}^{\mathrm{3}} \rho}{1.2 .3 \mathrm{~d} r^{3}} \Delta r^{3} \\
+\frac{\mathrm{d}^{4} \rho}{1.2 .3 .4 \mathrm{~d} r^{4}} \Delta r^{4}+\& \mathrm{c} .
\end{gathered}
$$

neglecting the sums of the odd terms*

$\mathfrak{m} \phi r \Delta r, \mathfrak{m} \psi r \Delta x^{2} \Delta r, \& c$.

we find equations of the form

$$
\begin{aligned}
& \frac{\mathrm{d}^{2} \xi}{\mathrm{d} t^{2}}=a^{2} \cos X \frac{\mathrm{d}^{3} g}{\mathrm{~d} r^{2}}+\mathfrak{a}^{2} \cos X \frac{\mathrm{d}^{4} \rho}{\mathrm{d} r^{4}} \\
& \frac{\mathrm{d}^{2} \eta}{\mathrm{d} t^{8}}=b^{2} \cos Y \frac{\mathrm{d}^{2} g}{\mathrm{~d} r^{2}}+\mathfrak{h}^{2} \cos Y \frac{\mathrm{d}^{4} \rho}{\mathrm{d} r^{4}} \\
& \frac{\mathrm{d}^{2} \zeta}{\mathrm{d} t^{2}}=c^{2} \cos z \frac{\mathrm{d}^{2} \rho}{\mathrm{d} r^{2}}+\mathfrak{c}^{2} \cos \boldsymbol{\mathrm { d } ^ { 4 } \rho} \frac{\mathrm{d} r^{4}}{2} \\
& a^{2}=\frac{\mathfrak{m}}{2} \Sigma\left\{\phi r+(\phi r) \Delta x^{2}\right\} \Delta r^{2} \\
& \mathfrak{a}^{2}=\frac{\mathfrak{m}}{24} \Sigma\left\{\phi r+(\psi r) \Delta x^{2}\right\} \Delta r^{4} \\
& b^{2}=\frac{\mathfrak{m}}{2} \Sigma\left\{\phi r+(\psi r) \Delta y^{2}\right\} \Delta r^{2} \\
& \mathfrak{h}^{2}=\frac{\mathfrak{m}}{24} \Sigma\left\{\phi r+(\psi r) \Delta y^{2}\right\} \Delta r^{4} \\
& c^{2}=\frac{\mathfrak{m}}{2} \Sigma\left\{\phi r+(\psi r) \Delta z^{2}\right\} \Delta r^{2} \\
& \mathfrak{c}^{2}=\frac{\mathfrak{m}}{24} \Sigma\left\{\phi r+(\psi r) \Delta z^{2}\right\} \Delta r^{4}
\end{aligned}
$$

$a^{2}, b^{2}, c^{2}$ being constant and the same as in Fresnel's notation.

- Mr. Kelland makes $\Sigma \varphi(r) \delta x^{2}=\Sigma \varphi(r) \delta y^{2}=\Sigma \varphi(r) \delta z^{2}$.

Cambridge Trans., vol. vi. p. 159.

But this $I$ apprehend is a different case from that contemplated by Fresnel. 
420 On the Wave-surface in the Theory of Double Refraction.

$$
\begin{aligned}
\frac{\mathrm{d}^{2} \rho}{\mathrm{d} t^{2}} & =\cos X \frac{\mathrm{d}^{2} \xi}{\mathrm{d} t^{2}}+\cos Y \frac{\mathrm{d}^{8} \eta}{\mathrm{d} t^{8}}+\cos z \frac{\mathrm{d}^{2} \zeta}{\mathrm{d} t^{8}} \\
\frac{\mathrm{d}^{2} \rho}{\mathrm{d} t^{2}} & =\left\{a^{2} \cos ^{2} X+b^{2} \cos ^{8} Y+c^{2} \cos ^{2} z\right\} \frac{\mathrm{d}^{2} \rho}{\mathrm{d} r^{9}} \\
& +\left\{\mathfrak{a}^{2} \cos ^{2} X+\mathfrak{b}^{8} \cos ^{2} Y+\mathfrak{c}^{2} \cos ^{2} z\right\} \frac{\mathrm{d}^{4} \xi}{\mathrm{d} r^{4}} \\
\text { If } \quad v^{2} & =a^{2} \cos ^{2} X+b^{2} \cos ^{8} Y+c^{2} \cos ^{8} z \\
\mathfrak{b}^{2} & =\mathfrak{a}^{2} \cos ^{2} X+\mathfrak{b}^{2} \cos ^{2} Y+\mathfrak{c}^{2} \cos ^{2} z \\
\frac{\mathrm{d}^{2} \rho}{\mathrm{d} t^{2}} & =v^{3} \frac{\mathrm{d}^{2} \rho}{\mathrm{d} r^{2}}+\mathfrak{v}^{2} \frac{\mathrm{d}^{4} \rho}{\mathrm{d} r^{4}} .
\end{aligned}
$$

This equation is readily integrable if $v$ and $\mathfrak{n}$ are constant, that is, if the direction of the displacement be invariable; and o may be expressed by a series of terms similar to

$(a \sin k r+b \cos k r) \sin n t+\left(a^{\prime} \sin k r+b^{\prime} \cos k r\right) \cos n t$, and represents a wave of light moving in the direction of $r$ with the velocity $\frac{n}{k}$,

$$
\frac{n}{k}=v\left\{1-\frac{k^{2}}{2.3 .4} \frac{\mathfrak{b}^{2}}{v^{2}}\right\}
$$

See Mr.Tovey's excellent paper, L.\&E. Phil. Mag. January 1836.

Fresnel does not implicitly take into consideration the term $\frac{\mathfrak{b}^{2} \mathrm{~d}^{4} \rho}{\mathrm{d} r^{4}}$, which according to M. Cauchy is necessary to explain dispersion. We have then

$$
\frac{\mathrm{d}^{2} \rho}{\mathrm{d} t^{2}}=v^{2} \frac{\mathrm{d}^{2} \rho}{\mathrm{d} r^{2}} \quad v=\frac{n}{k} .
$$

Fresnel supposes that the quantity $v^{8}$ will be constant when the force revolved in a direction perpendicular to the direction of displacement $\Delta \rho$ is also perpendicular to a given plane, and he shows that this will be the case precisely when the quantity $v^{8}=a^{3} \cos ^{2} X+b^{2} \cos ^{2} Y+c^{2} \cos ^{2} Z$ is a maximum or minimum. If the given plane is parallel to the plane

$$
n x+n y+l z=0 \text {. }
$$

Fresnel finds by geometry and without further assumptions the equation

$$
\begin{gathered}
\left(a^{2}-v^{2}\right)\left(c^{2}-v^{2}\right) n^{2}+\left(b^{2}-v^{2}\right)\left(c^{2}-v^{2}\right) m^{2} \\
+\left(a^{2}-v^{2}\right)\left(b^{2}-v^{2}\right) l^{2}=0 .
\end{gathered}
$$

The calculation of Fresnel's equation to the wave-surface is also now a purely geometrical problem which is easily effected by the elegant artifices suggested by $\mathrm{Mr}$. Smith in 
the vith volume of the Transactions of the Cambridge Philosophical Society, p. 85.

This equation to the wave-surface is

$$
\begin{gathered}
\left(x^{2}+y^{2}+z^{2}\right)\left(a^{2} x^{2}+b^{2} y^{2}+c^{2} z^{2}\right)-a^{2}\left(b^{2}+c^{2}\right) x^{2} \\
-b^{2}\left(a^{2}+c^{2}\right) y^{2}-c^{2}\left(a^{2}+b^{2}\right) z^{2}+a^{2} b^{2} c^{2}=0 .
\end{gathered}
$$

The coordinate axes being those of elasticity.

M. Cauchy has arrived at a similar equation, which is given at the foot of page 63, vol. v. of the Exercises. M. Cauchy seems to consider this equation identical with that of Fresnel. See Mémoires de l'Institut, tom. x. p. 312. See also Mr. Lloyd's excellent Report on Physical Optics, Fourth Report of the British Association, p. 391. In order that this may be the case I apprehend that it would be necessary to have $P$ in M. Cauchy's notation identical with $a^{2}$ in that of Fresnel. $Q$

$\boldsymbol{R}$ $b^{2}$

which I confess does not seem to me to have been proved. Moreover Fresnel's wave-surface depends upon the situation of certain fixed axes (of elasticity), while M. Cauchy's wavesurface does not appear to depend upon them in the same manner, and therefore $I$ suspect that the conclusions of that eminent mathematician are so far in discordance with those of Fresnel. I submit this opinion with great deference to those more conversant than myself with physical optics and with M. Cauchy's works, but at all events I think it will be admitted that the question is of sufficient importance to deserve further elucidation by showing, if it be possible, that M. Cauchy's quantities $P, Q, R$ have the same signification as Fresnel's $a^{2}, b^{\mathrm{a}}, c^{\mathrm{g}}$.

LV. On the Chemical Composition of Vegetable Membrane and Fibre*; with a Reply to the Objections of Professor Henslow and Professor Lindley. By the Rev. J. B. READE, M. $A$.

I $T$ is stated by Professor Henslow in his " Descriptive and 1 Physiological Botany ${ }^{\prime}$ "that all that is known of the chemical composition of the two elementary textures of plants, viz. membrane and fibre, has been derived from experiments made upon the gross material imperfectly separated from the various matters which the cells and tubes contain; that in this state the gross material is found to be composed of the

- Read before Section D, Zoology and Botany, of the Seventh Meeting of the British Association held at Liverpool; and now communicated by the Author.

+ Cabinet Cyclopredia. Henslow's Principles of Botany, p. 13. 